\title{
Las prácticas pre profesionales. Caso: Carrera de Educadores de Párvulos de la Universidad de Guayaquil
}

\section{Pre-professional practices. Case: Early Childhood Educators Race from the University of Guayaquil}

Lenin Mendieta, Angélica León, ${ }^{2}$ Carlos Idrovo ${ }^{3}$

\section{RESUMEN}

El objetivo fue determinar la incidencia de las prácticas pre profesionales en el desarrollo de las competencias de los estudiantes de la Carrera de Educadores de Párvulos. Fue un estudio exploratorio de campo, descriptivo, mixto: cuantitativo, cualitativo, correlacional.

La población fue de 84 sujetos, se realizó un consentimiento informado por escrito de participación, se realizó encuestas a los practicantes, se evaluaron las prácticas mediante una rúbrica de observación, se entrevistó a tutores y gestores. Los resultados de la encuesta a estudiantes determinaron que, un 25\% conocen que documentación es necesaria para cumplir con sus prácticas; el 14.3\% perciben que existe una comunicación regular entre el departamento de Prácticas Pre profesionales (PPP) con ellos; el 20.2\% perciben que existe mala calidad en el seguimiento que realizan los gestores de prácticas; el 23.8\% percibe que es mala la gestión sobre el acompañamiento de los tutores; un $13.1 \%$ evalúa deficiente el desempeño del Departamento de Prácticas Pre profesionales; un 64.3\% considera que las PPP ayudan muy poco a desarrollar competencias docentes.; Las rúbricas de evaluación determinaron que, los estudiantes realizan la programación de la actividad educativa en un $14.3 \%$ eficientemente; un $14.3 \%$ formula los objetivos eficientemente; en la selección y secuencia de contenidos de la programación de aula un 14.3\% lo hace de forma eficiente; el 14.3\% involucra eficientemente a los estudiantes en el proceso de aprendizaje; el 7.1\% maximiza el tiempo dedicado al aprendizaje de forma muy eficiente; el 14.3\% promueve el razonamiento, la creatividad y el pensamiento crítico.

En la evaluación del progreso de los aprendizajes para retroalimentar a los estudiantes y adecuar su enseñanza, el $14.3 \%$ es eficiente; propician un ambiente de respeto y proximidad en un $4.8 \%$ eficientemente; $y$, regulan positivamente el comportamiento de los estudiantes, el $4.8 \%$ de forma muy eficiente; en la entrevista a los docentes tutores de prácticas se determinó que existen discrepancias entre los criterios emitidos por los mismos. Las conclusiones a las que se llegó es que se debe diseñar un plan estratégico de prácticas pre profesionales, basado en competencias curriculares ecológicas siguiendo la filosofía de la Universidad de Guayaquil.

Palabras clave: practicas pre profesionales, competencias docentes curriculares, Educadores de párvulos

1.Universidad de Guayaquil. lenin.mendietat@ug.edu.ec 


\section{ABSTRACT}

The objective of this study was to determine the incidence of pre-professional practices in the development of the competencies of the students the pre-school. It was an exploratory, descriptive, interpretative, mixed quantitative, qualitative and correlational study.

The population consisted of 84 subjects, an informed consent of participation was carried out, practitioners were surveyed, practices were evaluated through an observation rubric, tutors and managers were interviewed. The results of the student survey determined that $25 \%$ know what documentation is necessary to comply with their practices; 14.3\% perceive that there is regular communication between the department of Pre-professional Practices (PPP) with them; $20.2 \%$ perceive that there is poor quality in the follow-up carried out by the practice managers; $23.8 \%$ perceive that the management on the accompaniment of the tutors is bad; $13.1 \%$ poorly assess the performance of the Department of Pre-professional Practices; $64.3 \%$ believe that these practices help very little to develop teaching skills. The evaluation rubrics determined that students do the programming/planning of the educational activity in a $14.3 \%$ efficiently; $14.3 \%$ formulates the objectives efficiently; In the selection and sequence of classroom programming content, $14.3 \%$ do so efficiently; $14.3 \%$ efficiently involve students in the learning process; $7.1 \%$ maximizes the time spent learning very efficiently; $14.3 \%$ promote reasoning, creativity and critical thinking.

In assessing, the learning progress to provide student's feedback and adapt their teaching, $14.3 \%$ is efficient; $4.8 \%$ promote an environment of respect and proximity efficiently; and $4.8 \%$ positively regulate student behaviour very efficiently. In the interview with the teacher tutors of practices, it was determined that there are discrepancies between the criteria issued by them. This study concludes that based on ecological curricular competences following the philosophy of Guayaquil University, a strategic plan of pre-professional practices must be designed.

Keywords: pre-professional practices, curricular teaching competencies, pre-school teachers.

\section{INTRODUCCIÓN}

Las prácticas son el periodo en que el estudiante se acerca a la realidad profesional y es el momento propicio para desarrollar y adquirir habilidades, destrezas y competencias generales y profesionales (Casillas, Cabezas, \& Serrate, 2017).

El llegar a ser docente es un proceso social e histórico que se va hilando de forma secuencial y progresiva; empieza antes de entrar a las aulas universitarias para formarse como docente, se entreteje con complejas interacciones entre las concepciones construidas desde la propia historia del sujeto, se da desde las primeras clases que se recibe en la escuela, transcurre por los pasillos, aulas y laboratorios del colegio, se forja en las aulas universitarias, fortaleciéndose con las prácticas pre profesionales como requisito para avanzar en ese recorrido que le permitirá obtener la profesión de docente, estas últimas, forman parte del entramado epistémico en la construcción del sujeto docente. Todo este proceso de construcción, se realiza dentro de un contexto social que es histórico y cultural.

Las prácticas pre profesionales, inciden en la identidad del futuro docente (García, González, \& Martín, 2016), para ello es necesario que en la formación sistemática de los futuros buenos docentes (Porta \& Sarasa, 2012; Porta \& Flores, 2014; Porta, De Lau rentis, \& Aguirre, 2015) se imbriquen dispositivos de revisión evaluativa de la calidad de la práctica pre profesional (Vilá, Aneas, \& Rajadell, 2015) como la planificación de un conjunto de clases, además, la revisión crítica del hábito profesional (en donde habiten el sistema de creencias del sujeto, sus requisitos previos sobre el objeto de conocimiento, las habilidades, destrezas y competencias que posee) que se va deconstruyendo en el practicante, además, los valores axiológicos que cohabitan en ellos y 
que son imbricados por sus docentes desde la relación educativa que existe.

\section{Planteamiento del Problema}

La Asamblea Nacional del Ecuador (2008) en su artículo 27 sustenta la voluntad del estado de garantizar una educación holística, integral, incluyente y crítica; además el artículo 350 pone de manifiesto que la educación superior tiene como fin la formación profesional desde una visión científica y humanista; sumado a estos, se encuentra la Ley Orgánica de Educación Superior (LOES) que permite interpretar en su artículo 93 que para que la educación sea de calidad es preciso que se establezcan relaciones entre todas las partes que conforman esta estructura, así, se conmina a que docentes y estudiantes construyan una cultura de calidad educativa, desde la investigación e innovación y la vinculación con la sociedad (LOES, 2018).

Esta invitación es realizada desde la visión de un horizonte de ciencia y saberes ancestrales, de valores morales y principios de vida universales. El Consejo de Acreditación de la Calidad Educativa Superior (CACES) es el órgano encargado de supervisar, evaluar y acreditar esa calidad educativa (CACES, 2018).

La UNESCO (2018) adopta una firme postura en cuanto a sus políticas, señala que las competencias que adquiere el sujeto que aprende, le serán fundamentales en su campo laboral, la misma coadyuvará a la disminución de la pobreza y un desarrollo sostenible de los pueblos, argumenta que debe existir para el 2030 el acceso igualitario a la educación de calidad, sea esta técnica superior o profesional universitaria. Sobre las Instituciones de Educación Superior en Ecuador.

El problema que se presenta en la Facultad de Filosofía, Letras y Ciencias de la Educación de la Universidad de Guayaquil, específicamente en la Carrera de Educadores de Párvulos, es que los estudiantes desarrollan sus habilidades, destrezas y competencias de forma un tanto ortodoxa, en algunas ocasiones, estas se trabajan desde la generalización de los conceptos de su campo de formación profesional sin que exista un plan de desarrollo de estas prácticas (Huachisaca, 2017), con lo cual, el empoderamiento de la teoría se queda sin soporte práctico.

Dentro de los perfiles de egreso de los estudiantes de la carrera objeto de estudio, se encuentra el trabajar con niños de educación inicial que va desde los tres hasta los cinco años, a pesar de ello, las prácticas pre profesionales son realizadas en algunas ocasiones hasta en Cuarto año de Educación General Básica con niños de diez años.

El contexto en donde se desarrolla la práctica pre profesional debe ser en centros educativos, a pesar de ello, en muchas ocasiones estas se realizan en comunidades barriales con niños que no están insertos en el régimen escolar, con lo cual, la planificación se aleja del constructo curricular nacional, teniendo que extrapolar los conocimientos sobre educación regular. Estas prácticas fuera del campo específico de la educación inicial, dislocan el vector metodológico de la planificación didáctica convirtiéndose en planes de guardería, cuidados a niños con discapacidades entre otros.

Por lo expuesto, se planteó el problema y pregunta de investigación señalando:

¿La incidencia de las prácticas pre profesionales determinan el desarrollo de las competencias de los estudiantes de la Carrera de Educadores de Párvulos en el periodo lectivo 2018-2019?

¿Las prácticas pre profesionales, inciden en el desarrollo de las competencias de los estudiantes de la Carrera de Educadores de Párvulos en el periodo lectivo 2018-2019?

Se establece como las prácticas pre profesionales determinan el desarrollo de las competencias de los estudiantes de la Carrera de Educadores de Párvulos en el periodo lectivo 2018-2019 


\section{Objetivo General}

Determinar la incidencia de las prácticas pre profesionales en el desarrollo de las competencias de los estudiantes de la Carrera de Educadores de Párvulos en el periodo lectivo 2018-2019

\section{MÉTODOS Y MATERIALES}

Fue un estudio exploratorio de campo, descriptivo, mixto: cuantitativo y cualitativo, interpretativo y correlacional. Se buscó determinar la incidencia de las prácticas pre profesionales en el desarrollo de las competencias de los futuros docentes de la Carrera de Educadores de Párvulos en el periodo lectivo 2018-2019. La población de 84 estudiantes ( 80 mujeres y 4 hombres) los cuales realizaron sus prácticas pre profesionales, se codificó a la población (donde 0 fue el código para Otros, 1 para Femenino y 2 para Masculino).

Fue un grupo etario con rango de edad de entre 21 y 27 años. Además, como parte de la investigación se tomó en consideración a cuatro tutores y un gestor de prácticas pre profesionales, esto sumó un total de 89 sujetos.

Se realizó un consentimiento informado por escrito para los sujetos a estudio, se explicó detalladamente, el objetivo, metodología y fines de la investigación, quedó sentado entre otras: el deseo de participar en la investigación y la libertad de salir de la misma cuando ellos así consideren oportuno, se tomaron muy en cuenta las normas éticas de la investigación educativa y bioética (Galeano, 2004; Sañudo, 2006; Asociación Médica Mundial, 2017).

Para conocer de cerca los procesos de PPP, se realizó el análisis de las normativas y reglamentos en un estudio asincrónico comparado (Villalobos, 2002), tomando como data el periodo comprendido entre 2009-2019, se revisó los documentos oficiales de la Carrera Educadores de Párvulos.
Se utilizaron técnicas de encuesta a los estudiantes (Bernal, 2006; Behar, 2008), se diseñó un instrumento con preguntas semiestructuradas. Se entrevistó a los tutores y gestores de prácticas en el periodo lectivo 2018-2019, para este fin se diseñó un guion de entrevista para cada grupo.

Los instrumentos de encuesta fueron validados por pares ciegos externos: además se realizó una encuesta piloto en la Carrera de Educación Inicial en el mismo semestre de realización de estas prácticas pre profesionales para validar las mismas.

Los sujetos entrevistados validaron la entrevista en dos momentos: un primer momento, cuando se presentó el instrumento de entrevista para que ellos determinen la pertinencia de las preguntas; el segundo momento: cuando les presentamos el documento desgravado con las respuestas que ellos emitieron, para que puedan reafirmar o retractarse.

Se evaluaron las prácticas PPP del año lectivo 2018-2019; para ello se construyó una rúbrica de evaluación de observación, la misma contó con criterios y parámetros pertinentes a las normativas y reglamentos vigentes en la Carrera Educadores de Párvulos.

El diseño de la rúbrica abarcó el contexto carrera, las competencias genéricas de la Universidad de Guayaquil y las específicas de la carrera, el perfil de egreso que tienen los estudiantes de la carrera, los objetivos de los programas y sus contenidos; además los resultados de los aprendizajes para establecer los criterios de evaluación y otorgar pertinencia a la evaluación (Andrade \& Ying, 2005; Kocakülah, 2010), también, se adaptaron criterios de evaluación de las prácticas pre profesionales que se consideró saludables para la mejora de la calidad de la educación desde ese contexto (Álvarez, 2000; Ramírez Alfaro, 2001). 


\section{RESULTADOS}

Encuesta a estudiantes

1. ¿A qué tipo de Género Sexual pertenece?

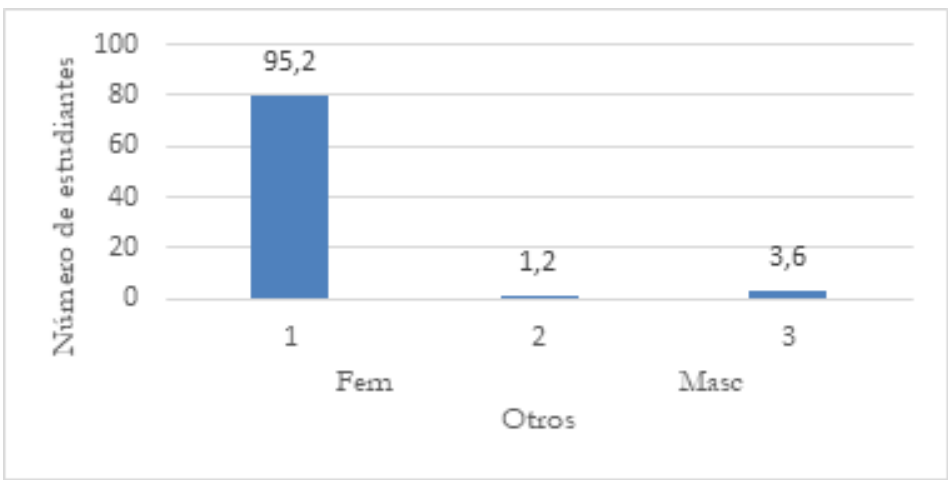

2. ¿Actualmente trabaja como docente?

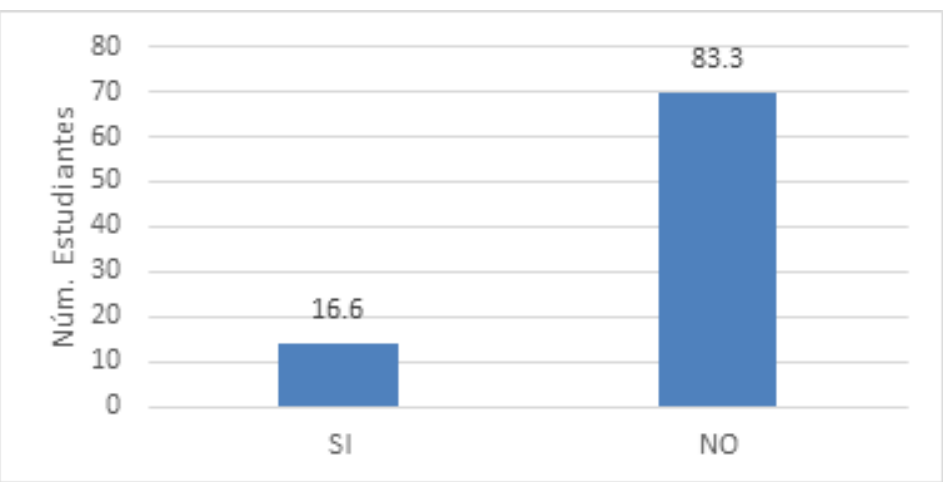

3. ¿Antes de realizar la práctica pre profesional Le dieron información de cuándo podría empezar a realizarse?

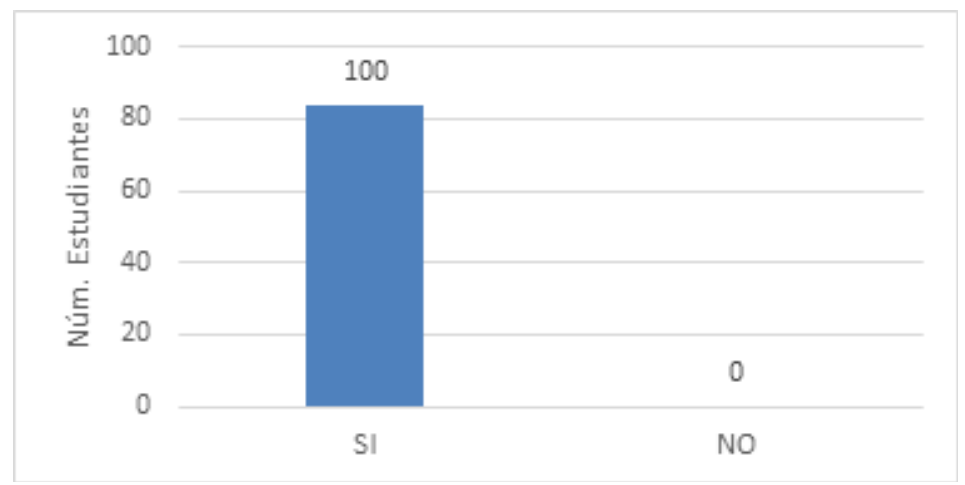


4. ¿Conoce cuál es la documentación que se debe presentar para cumplir con las prácticas pre profesionales?

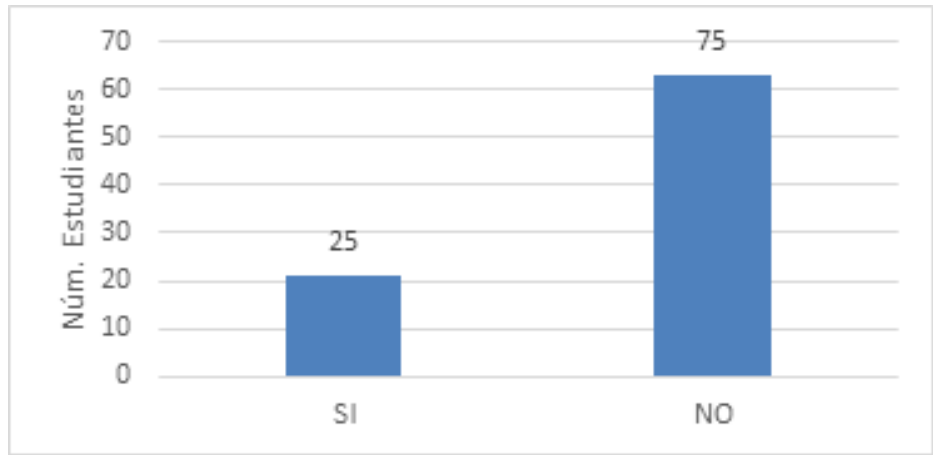

5. ¿Tiene a disposición alguna institución educativa para realizar las prácticas pre profesionales?

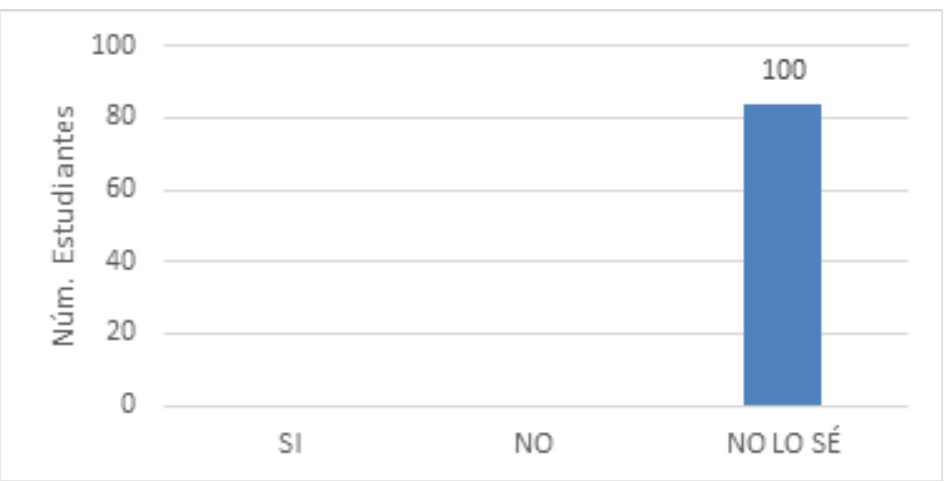

6. ¿Cuál es su percepción sobre la comunicación que existe entre el Departamento de Prácticas con los estudiantes de la carrera?

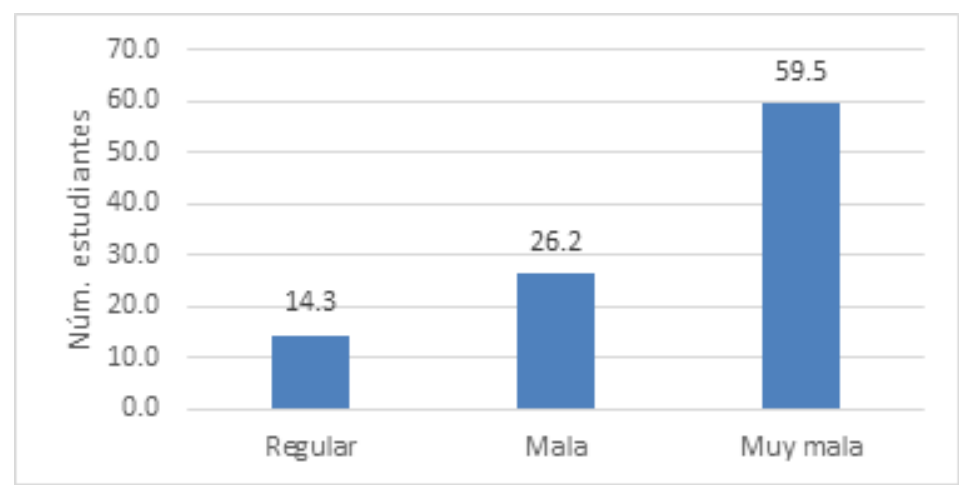

7. ¿Cuán es su percepción sobre la calidad en el seguimiento que realizan los docentes gestores a las prácticas pre profesionales?

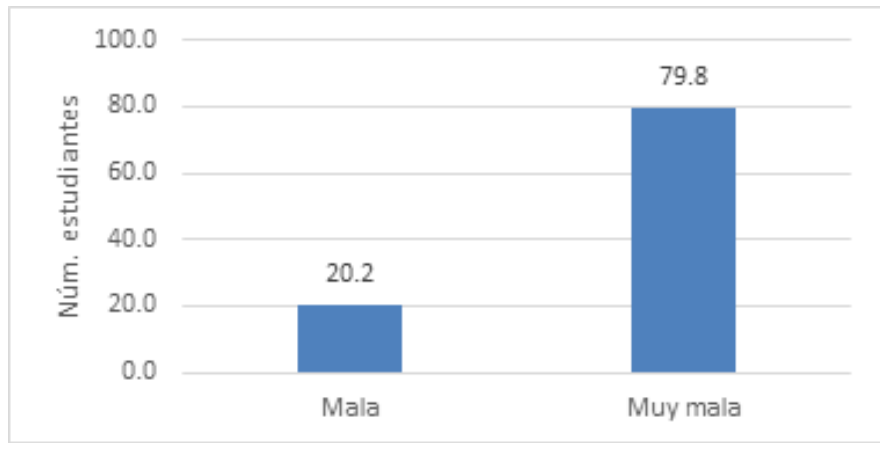


8. ¿Cuál es tu percepción del acompañamiento y tutorías que realizan sus docentes tutores a sus planificaciones?

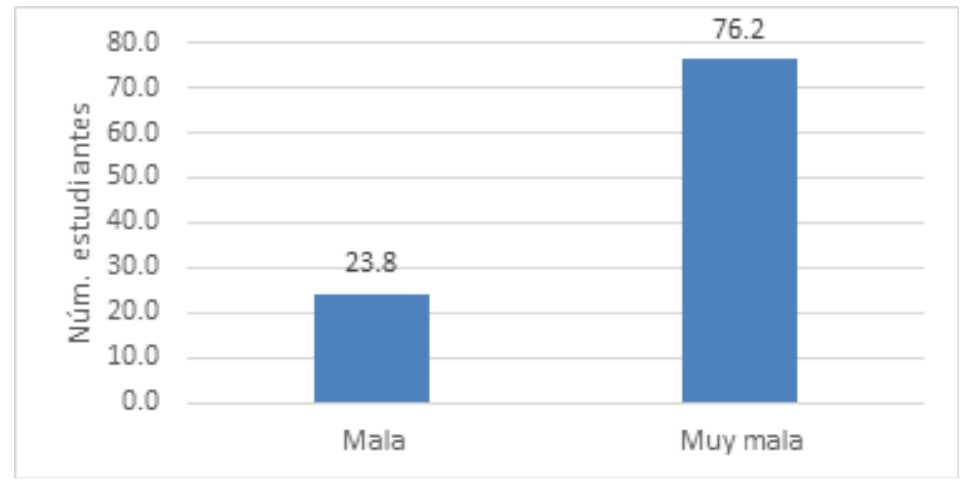

9. ¿Cómo evalúa el desempeño del trabajo que se realiza en el departamento de prácticas?

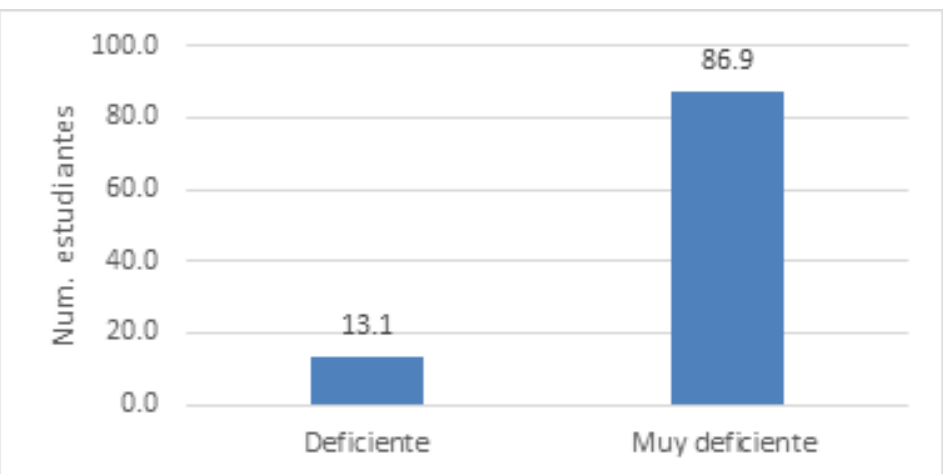

10. ¿Consideras que, las prácticas pre profesionales le ayudan a desarrollar competencias para la docencia?

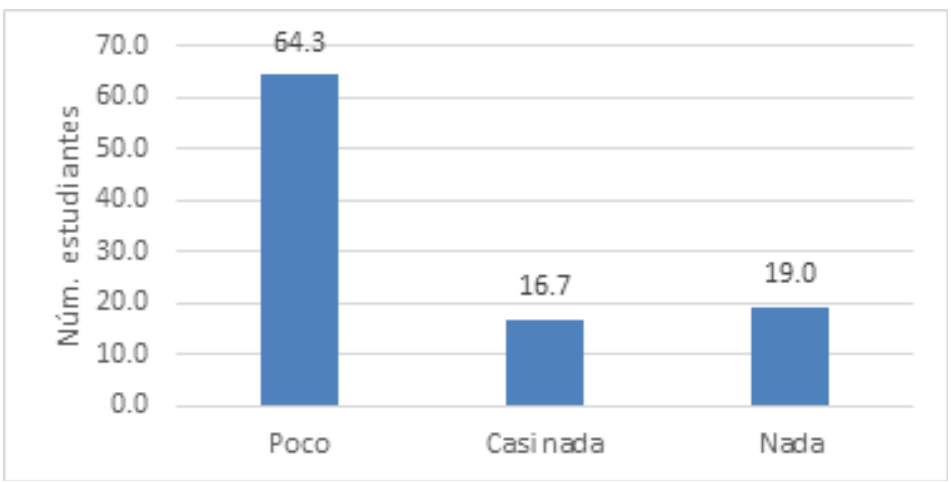




\section{Rúbrica de evaluación a la PPP}

1.Realiza la programación de la actividad educativa teniendo como referencia el currículo y las unidades didácticas, además; instrumentos de planificación que conoce y utiliza.

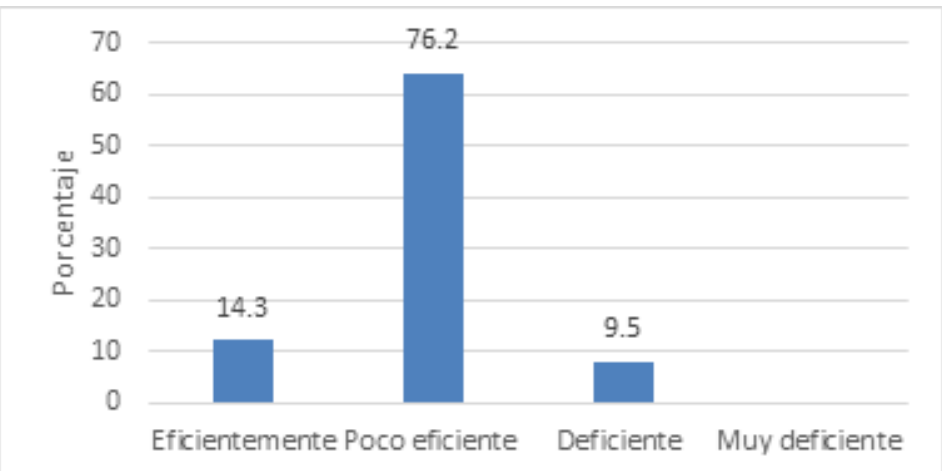

2. Formula los objetivos didácticos de forma que expresan claramente las habilidades que los alumnos deben conseguir como reflejo y manifestación de la intervención educativa.

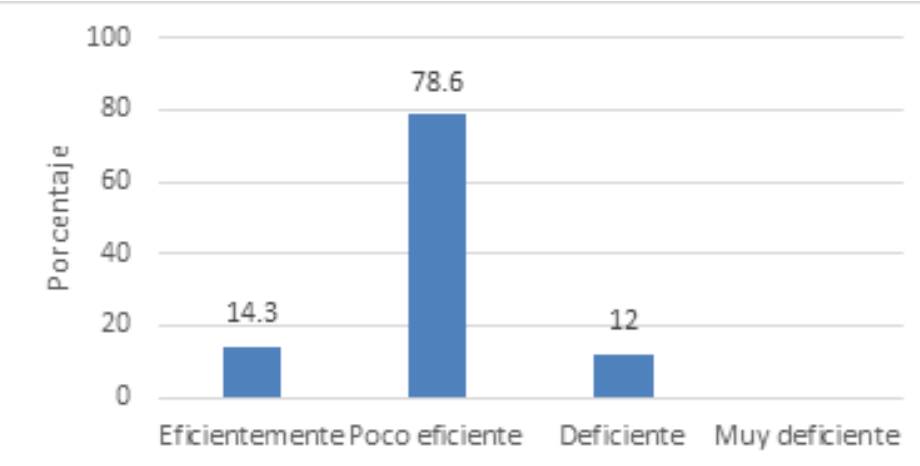

3. Selecciona y secuencia los contenidos (conocimientos, procedimientos y actitudes) de la programación de aula con una distribución y una progresión adecuada a las características de cada grupo de alumnos.

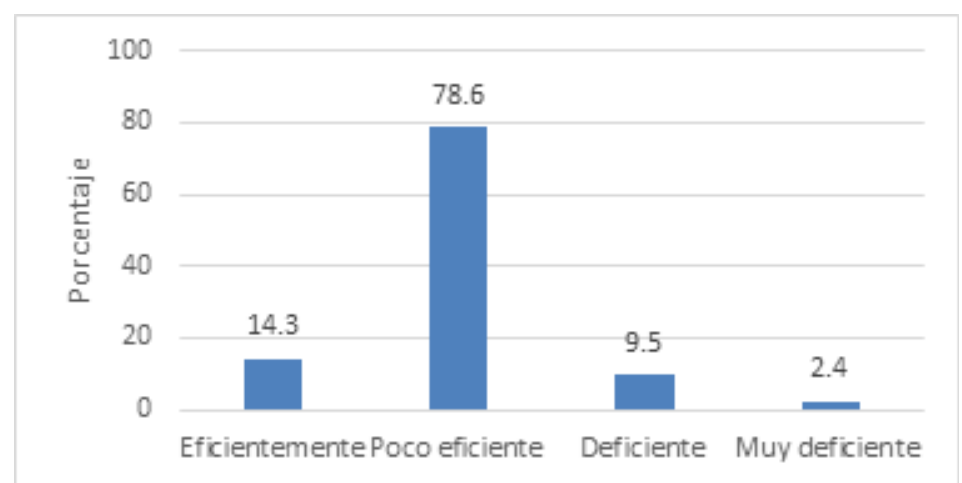


4. Involucra activamente a los estudiantes en el proceso de aprendizaje: Logra la participación activa y el interés de los/as estudiantes por las actividades propuestas, ayudándolos a ser conscientes del sentido, importancia o utilidad de lo que se aprende

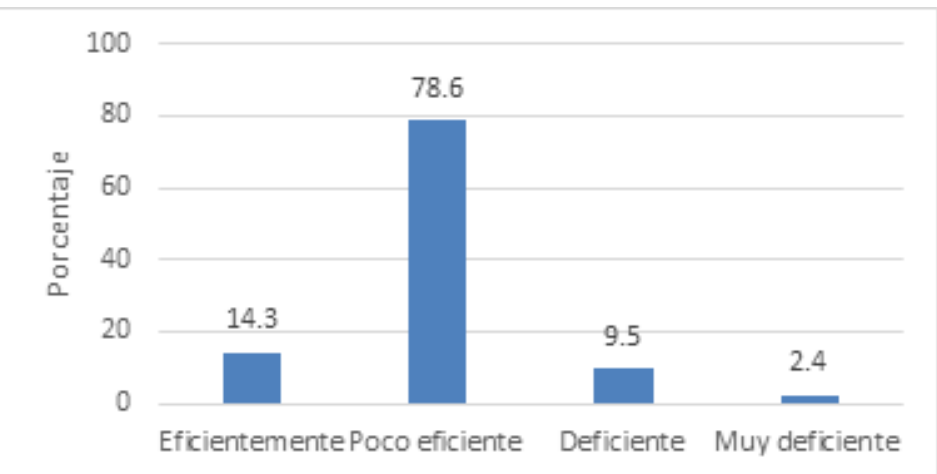

5. Maximiza el tiempo dedicado al aprendizaje: Usa de manera efectiva el tiempo, logrando que, durante toda o casi toda la sesión los estudiantes estén ocupados en actividades de aprendizaje.

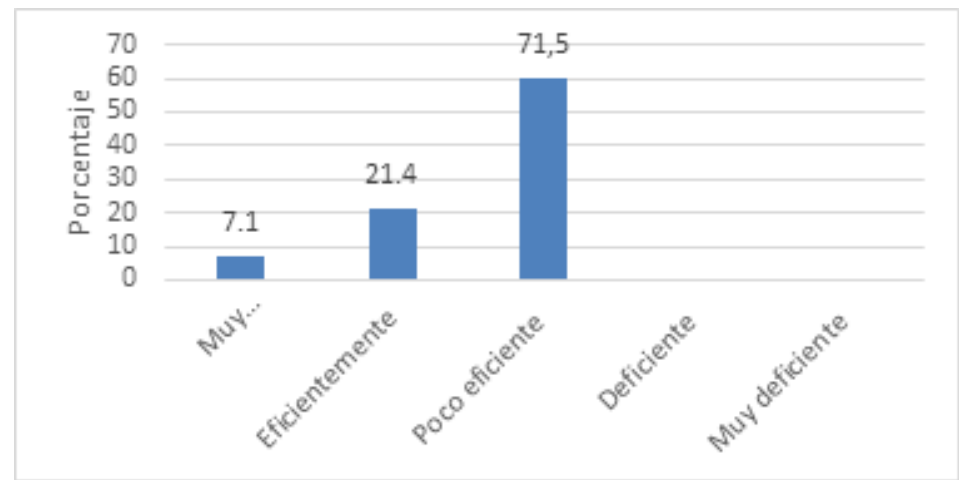

6. Promueve el razonamiento, la creatividad y/o el pensamiento crítico: Propone actividades de aprendizaje y establece interacciones pedagógicas que estimulan la formulación creativa de ideas o productos propios, la comprensión de principios, el establecimiento de relaciones conceptuales o el desarrollo de estrategias

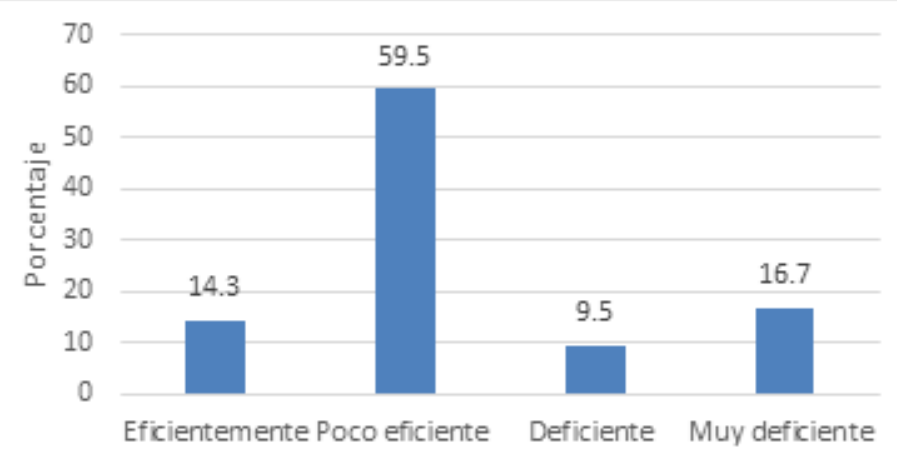


7. Evalúa el progreso de los aprendizajes para retroalimentar a los estudiantes y adecuar su enseñanza: Acompaña el proceso de aprendizaje de los/as estudiantes, monitoreando sus avances y dificultades en el logro de los aprendizajes esperados en la sesión y, a partir de esto, les brinda retroalimentación formativa y/o adecúa las actividades de la sesión a las necesidades de aprendizaje identificadas.

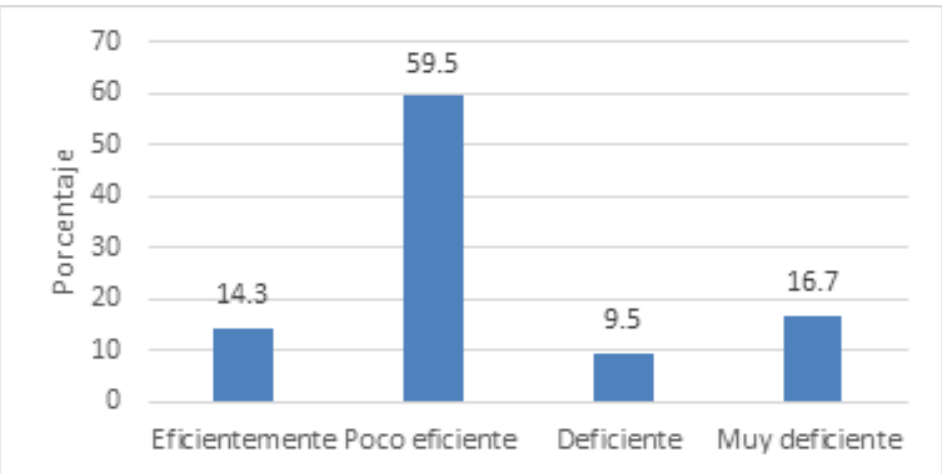

8. Propicia un ambiente de respeto y proximidad: Se comunica de manera respetuosa con los/as estudiantes y les transmite calidez o cordialidad dentro del aula. Además, está atento y es sensible a sus necesidades afectivas o físicas, identificándolas y respondiendo a ellas con comprensión y empatía

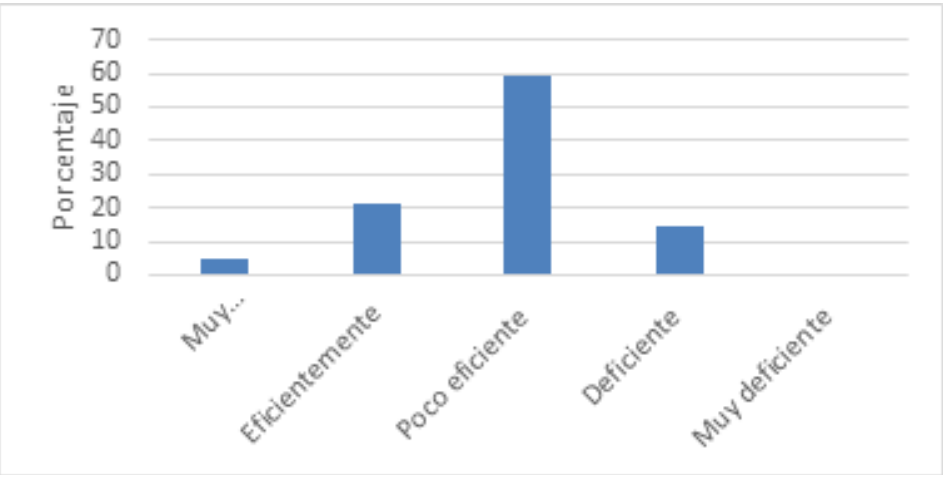

9. Regula positivamente el comportamiento de los estudiantes: Las expectativas de comportamiento o normas de convivencia son claras para los/as estudiantes. El/la docente previene el comportamiento inapropiado o lo redirige eficazmente a través de mecanismos positivos que favorecen el buen comportamiento y permiten que la sesión se desarrolle sin mayores contratiempos

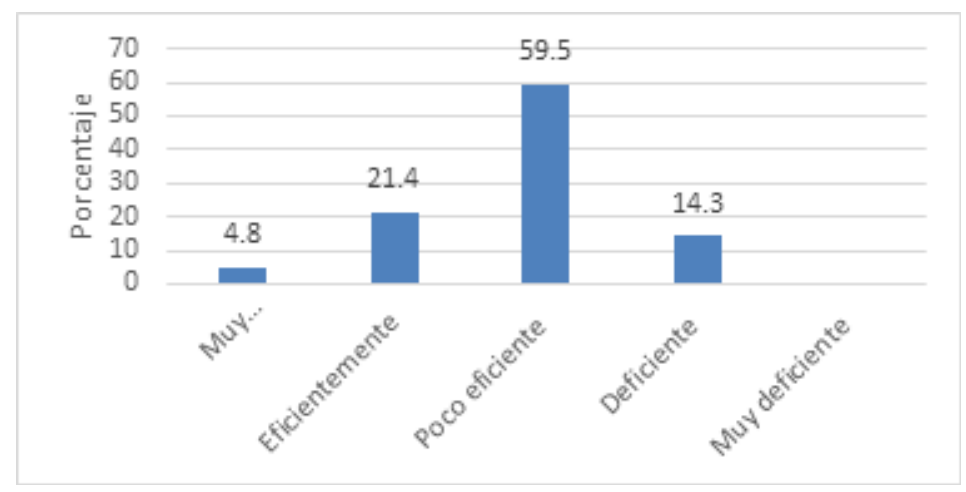




\section{Entrevistas a tutores}

En la entrevista realizada a los tutores existió pertinencia en los criterios emitidos por los mismos, se interpreta y caracteriza las entrevistas en base a las preguntas formuladas:

1.¿Cuál es el objetivo fundamental de una práctica pre profesional en la Carrera de Educadores de Párvulos?

Los docentes aseveran que de lo que se trata es de consolidar (mediante situaciones reales) la formación recibida del estudiante como futuro profesional de la docencia parvularia, para afirmar su saber profesional como una síntesis reflexiva y crítica de la teoría y práctica educativa

2.¿Cuál es el rol que desempeña como tutor de las PPP de sus estudiantes?

Los profesionales tutores jugamos (manifiestan los entrevistados) un papel fundamental en la formación de nuestros estudiantes, somos la primera imagen del mundo profesional que reciben. Es una relación mediante la cual el alumno entra en contacto con la función docente y sus diferentes formas de trabajar. En definitiva, el estudiante conocerá un estilo y un modelo profesional a través de nosotros como guías.

3.¿Cuáles son las estrategias que utilizan para el conocimiento del objeto y acompañamiento a sus estudiantes que realizan las prácticas dentro de su proceso de formación?

Lo primero que realizamos es una evaluación diagnóstica para conocer el perfil del estudiante, sus conocimientos, sus necesidades y aspiraciones, sus expectativas y temores, y su manera de aprender. Una vez encontrado ese perfil, se diseña una ruta metodológica personalizada la cual se la transita en todo momento junto al estudiante, se realizan fichas de seguimiento y se informa de los casos particulares a la gestoría de PPP cuando se presentan inconvenientes.
4.¿De qué manera realizan la comunicación entre la institución educativa en donde van a realizar las PPP y sus estudiantes?

La comunicación siempre es fluida, es realizada en la metodología de "relación a tres bandas", la cual consiste en un dialogo permanente entre el tutor-estudiante-profesor de aula, esto se lo realiza semanalmente en reuniones de trabajo para determinar cómo avanzan las prácticas y en que tiene más dificultades los practicantes. Esta relación a tres bandas, es pactada en base a cumplir objetivos de conocimiento, desarrollar y/o fortalecer actitudes, valores, habilidades, procedimientos y competencias que el practicante debe alcanzar.

5.¿Cómo diseñan el seguimiento de las PPP de sus estudiantes?

Los docentes manifiestan que es un diseño que se sostiene en la estructura:

a. Tiempo-espacio. - Es fundamental dotar del suficiente tiempo-créditos y el tener el espacio físico- áulico adecuado para la realización de las prácticas a los estudiantes, además de las suficientes horas para la supervisión y seguimiento a sus prácticas, esto da lugar a una mejor fluidez en la comunicación tutor-estudiante-docente de la institución, convirtiéndose en ese momento en prácticas significativas.

b. Objetivos.- Los objetivos del seguimiento a las prácticas son velar porque el estudiante tenga las condiciones necesarias para desarrollar su experiencia de profesionalización en tres áreas determinadas: el conocimiento del objeto respecto al ámbito específico de sus prácticas, el contexto institucional, el encargo social que tiene el docente como profesional, los recursos y materiales didácticos y la documentación; las habilidades, destrezas, competencias y procedimientos en torno a las relaciones de comunicación, escucha y negociación, técnicas de observación de sus clases, entrevistas personalizadas con los alumnos a su cargo, reuniones con sus pares, gestiones 
docentes, identificaciones de situaciones emergentes en el aula de clase, rendimiento de los alumnos a su cargo, cumplimiento de las planificaciones; $y$, actitudes y valores como autonomía para trabajar las clases, respeto, tolerancia, saber estar y todas estas cobijadas por la ética y la moral

c. Evaluación. - práctica-reflexión-práctica: observación de las prácticas, dialogo reflexivo sobre el desempeño de sus prácticas, determinación en conjunto de las fortalezas y debilidades observadas, acuerdos y compromisos de mejoramiento, observación de sus prácticas

d. Informe. - se presenta un informe de las prácticas de forma general e individual en donde se destaca mediante una ficha de seguimiento de aprendizajes experienciales de los estudiantes

\section{Entrevista a gestor de PPP}

En la entrevista al gestor de las PPP este asegura que, es la finalidad el certificar las horas de prácticas pre profesionales realizadas por los estudiantes, esta certificación es validada por un cuaderno de prácticas la cual tienen las firmas de los profesores de aula, los tutores de las prácticas, la dirección de la carrera y finalmente la gestoría de PPP, solo así se asegura el cumplimiento de estas actividades, las mismas son orientadas a la aplicación de conocimiento y al desarrollo de destrezas y habilidades específicas y a la adquisición de competencias que un estudiante debe adquirir para un adecuado desempeño en su futura profesión. Asegura, además., que, se realiza un seguimiento desde esa gestoría a cada carrera de la facultad para que se cumplan con las directrices establecidas

\section{CONCLUSIONES}

Con los datos obtenidos, se emitieron conclusiones desde una cosmovisión multidimensional, puesto que se aborda el estudio desde distintas aristas que permitieron tener un horizonte real y sin dislocaciones. Se concluye que los estudiantes que realizan PPP presentan deficiencias en cuanto al conocimiento de las normativas necesarias para la realización de sus PPP, existiendo una percepción negativa en cuanto al desempeño de los tutores y gestores de las PPP en la carrera de Educadores de Párvulos, en lo que se relaciona a la comunicación, seguimiento y tutorías; lo más alarmante es que los estudiantes consideran que las PPP ayudan muy poco a desarrollar competencias docentes.

Son pocos los estudiantes de PPP que realizan eficientemente la selección y secuencia de contenidos de la programación de aula, planificación y planteamiento de los objetivos de las planificaciones; así también son pocos los que se involucran eficientemente en el proceso de aprendizaje, debido a que no maximizan el tiempo que se debe dedicar a esos aprendizajes y no promueven el razonamiento, la creatividad y el pensamiento crítico, y, propician un ambiente de buen comportamiento, respeto y proximidad de forma poco eficiente.

El objetivo de las PPP es una buena formación como futuro profesional parvulario, que asiente su saber profesional y humano, es por ello que el rol que ejerza el tutor de PPP es fundamental ya que se constituye en la primera imagen del mundo profesional que tiene el estudiante; para ello, es preciso implementar estrategias que coadyuven a la adquisición del objeto científico, el desarrollo de habilidades, destrezas y competencias y, la adquisición y fortalecimiento de valores axiológicos. Es por ello que los tutores de esas prácticas siguen de cerca la evolución de sus estudiantes mediante evaluaciones de corte práctico-reflexivo-práctico, estableciendo acuerdos y compromisos con sus estudiantes, luego de lo cual presentan un informe general e individual de dichas prácticas.

Se debe diseñar un plan estratégico de prácticas pre profesionales, basado en competencias curriculares ecológicas siguiendo la filosofía de la Universidad de Guayaquil, que contribuya en la preparación de los estudiantes para la vida labo- 
ral y humana para que los estudiantes que realicen sus prácticas pre profesionales, lo hagan desde una vera en donde su contexto histórico social no sufra divorcios con la realidad laboral nacional, regional y mundial (Porta \& Sarasa, 2012; Porta \& Flores, 2014; Porta, De Lau rentis, \& Aguirre, 2015), ya que finalmente, de lo que se trata es de mejorar la calidad educativa (Vilá, Aneas, \& Rajadell, 2015).

\section{REFERENCIAS BIBLIOGRÁFICAS}

Anushek, E. (2005). ¿Por qué es importante la calidad de la educación? Finanzas \& Desarrollo, 15-19. Obtenido de https://goo.gl/sWUNyT

Asamblea Nacional del Ecuador. (20 de octubre de 2008). Constitución la República del Ecuador. Constitución de la República del Ecuador, 82. Quito, Ecuador. Recuperado el 12 de julio de 2018, de Constitución - Asamblea Nacional del Ecuador: https://goo.gl/wvoAoB

ASOCIACIÓN MÉDICA MUNDIAL. (21 de Marzo de 2017). Declaración de Helsinki de la AMM - Principios éticos para las investigaciones médicas en seres humanos, electrónica. Recuperado el 1 de febrero de 2017, de Declaración de Helsinki de la AMM - Principios éticos para las investigaciones médicas en seres humanos: https://goo. $\mathrm{gl} / 4 \mathrm{SnXoh}$

Boixader, A. (2010). Presentación. En J. Mélich, A. Boixader, M. Alguacil, j. Canelles, M. Fons, J. Llovet, ... A. Vicente, Los máregenes de la moral. Una mirada dética a la educación (primera ed., pág. 145 (16)). Barcelona: GRAÓ. doi:47K-M-7K27WM<

CACES. (2 de AGOSTO de 2018). Consejo de Evaluación, Acreditación y. Aseguramiento de la Calidad. Recuperado el 5 de JULIO de 2019, de sUPLEMENTO 298: http://cort. as/-L- $\mathrm{xE}$

Casillas, S., Cabezas, M., \& Serrate, S. (junio-abril de 2017). Evaluación de competencias: retos en la formación práctica de los pedagogos. Avaliação, 9(25), 90-109 (90). Recuperado el 5 de julio de 2019, de http://cort.as/-L_w3

García, S., González, R., \& Martín, A. (2016). Influencia de las prácticas en el desarrollo de la identidad profesional de los estudiantes. Pedagogía Social, 245-259 (245). doi:ISSN: 1139-1723; DOI: 10.SE7179/PSRI_2016.28.18

Hernández Sampieri, R., Fernández Collado, C., \& Baptista Lucio, M. d. (2014). Metodología de la investigación (sexta ed.). México: McGRAW-HILL. doi:ISBN: 978-1-45622396-0
Huachisaca, J. (2017). Manual de procesos y procedimientos de gestión de prácticas pre-profesionales. Guayaquil: Universidad de Guayaquil.

Kocakülah, M. S. (2010). Development and Application of a Rubric for Evaluating Students' Performance on Newton's Laws of Motion. Journal of Science Education and Technology, 19(2), pp 146-164. Recuperado el 4 de agosto de 2019, de https://n9.cl/icl9

Larrea, E. (2015). Modelo Curricular Ecologico Para la Educacion Superior con enfoque Ecologico. Guayaquil. Recuperado el 5 de julio de 2019

LOES. (DICIEMBRE de 12 de 2018). LEY ORGANICA DE EDUCACION SUPERIOR, LOES. Recuperado el 22 de mayo de 2017, de LEY ORGANICA DE EDUCACION SUPERIOR, LOES: aka-cdn.uce.edu.ec/ares/tmp/Elecciones/2\%20LOES.pdf

Porta, L., \& Flores, G. (2014). Las prácticas de enseñanza de profesores universitarios memorables. El estudiante como par antropológico. Journal for Educators, Teachers and Trainers JETT, 60-73. doi:ISSN 1989 - 9572

Porta, L., \& Sarasa, M. C. (2012). CONCEPCIONES DE LA BUENA ENSENANZA EN LOS RELATOS DOCENTES: LA FORMACION INICIAL DEL PROFESORADO DE INGLES. Praxis Educativa, 10(10), 68-74. Recuperado el 12 de agosto de 2018, de https://goo.gl/Zxg8Nm

Porta, L., De Lau rentis, C., \& Aguirre, J. (2015). Indagación narrativa y formación del profesorado: nuevas posibilidades de ruptura y construcción en la identidad docente. Praxis educativa, 43-49. doi:ISSN: 0328-9702

Sañudo, L. (2006). La ética en la investigación educativa. HALLAZGOS(6), 83-98. doi:ISSN: 1794-3841

UNESCO. (4 de junio de 2018). Educación y formación técnica y profesional (EFTP) - Unesco. (Oficina Regional de Educación para América Latina y el Caribe) Recuperado el 5 de Julio de 2019, de OFICINA DE LA UNESCO EN SANTIAGO:http://www.unesco.org/new/es/santiago/ education/technical-vocational-education-and-training/

Vargas, T., \& Mendieta, L. (2018). Descolonizar la docencia Universitaria Ecuatoriana. Polo del Conocimiento, 630654. doi:https://doi.org/10.23857/pocaip

Vilá, R., Aneas, A., \& Rajadell, N. (2015). La evaluación de competencias del alumnado en las Prácticas Externas. La perspectiva de todos los agentes implicados en las Prácticas Externas del grado de Pedagogía de la Universidad de Barcelona. PROCEDIA - Social and Behavioral Sciences, 226 - 232. doi:10.1016/j.sbspro.2015.07.034 